\title{
3D Air Filtration Modeling for Nanofiber Based Filters in the Ultrafine Particle Size Range
}

\author{
Wannes Sambaer ${ }^{\mathrm{a}}$, Martin Zatloukal ${ }^{\mathrm{a}}$ and Dusan Kimmer ${ }^{\mathrm{b}}$ \\ ${ }^{a}$ Centre of Polymer Systems, Polymer Centre, Tomas Bata University in Zlin, \\ nam. T. G. Masaryka 5555, 76001 Zlin, Czech Republic \\ ${ }^{b}$ SPUR a.s., T. Bati 299, 76422 Zlin, Czech Republic
}

\begin{abstract}
In this work, novel 3D filtration model for nanofiber based filters has been proposed and tested. For the model validation purposes, filtration efficiency characteristics of two different polyurethane nanofiber based structures (prepared by the electrospinning process) were determined experimentally in the ultrafine particle size range $(20-400 \mathrm{~nm})$. It has been found that the proposed model is able to reasonably predict the measured filtration efficiency curves for both tested samples.
\end{abstract}

Keywords: Nanofiber, Electrospinning, 3D Simulation, Filtration modeling.

PACS: 81.07.-b

\section{INTRODUCTION}

Nanofibers based nonwovens are widely applied in different areas such as filter media, cosmetic skin care, life science, nanosensors, tissue engineering, personal protective clothing and other medical and industrial applications [1-4].

Filter media is one of the largest application groups of nanofiber nonwovens because they are a good medium to produce high quality filters due to their high surface area and lower resistance to airflow. In order to optimize the nanofiber based filters as well as to understand the complicated link between their morphological characteristics (such as fiber diameter distribution/pore size distribution) and particle-fiber interactions with respect to filtration efficiency, the realistic 3D filtration modeling can be utilized. The main disadvantage of the currently utilized $3 \mathrm{D}$ filtration models is the fact that virtual rather than realistic morphological characteristics of the nanofiber based filters are taken into account [5-7]. In order to overcome this difficulty, a new 3D filtration model has been proposed in this work. For the model validation purposes, two different polyurethane (PU) nanofiber based filters have been produced by the electrospinning process and their filtration efficiency characteristics have been determined experimentally in ultrafine particle size range (20-400 nm). In the second part of the study, the effect of the particle-fiber interactions by using the proposed model has been investigated theoretically. 


\section{EXPERIMENTAL}

\section{Material and Nanofiber Nonwovens}

Polyurethanes are segmented polyadducts prepared from diisocyanate, polymer diol (soft segment) and chain extender (low molecular diol). Two different polyurethane solutions were used to create two types of nanofiber based nonwovens. The first polymer solution was prepared in dimethylformamide (DMF) from aromatic diisocyanate (4,4'methylenebis (phenylisocyanate), polyester diol (poly(3-methyl-1,5pentanediol)-alt-(adipic, isophtalic acid) and 1,4 butanediol and is referred in this work as 'solution $A$ '. The second polymer solution in DMF was based on aliphatic diisocyanate and polyether diol (HydroMed D640 from AdvanSource Biomaterials Corporation, MA, USA) and is referred as 'solution $B$ '. The prepared solutions were suitable for electrospinning and had a PU concentration of $13.8 \mathrm{wt}$. \%, for solution A and 16.2 wt. $\%$ for solution $\mathrm{B}$, viscosities of $1.5 \mathrm{~Pa} . \mathrm{s}$ and conductivities of $150 \mu \mathrm{S} . \mathrm{cm}^{-1}$.

Based on these two solutions two nanofiber structures were created by electrospinning process. The first nonwoven was based on four electrospun layers of solution A above each other and the latter nonwoven was a combination of one electrospun layer of solution A and one layer of solution B. The nanolayer prepared from solution A was more hydrophobic then the nanostructure prepared from solution B. This is why PU nanofibers prepared from solution A are referred as hydrophobic and PU nanofibers prepared from solution B are referred as hydrophilic ones. Both nonwovens (Sample 1 and Sample 2) have a similar mass area, 0.438 g.m ${ }^{-2}$ and $0.447 \mathrm{~g} . \mathrm{m}^{-2}$, respectively. The thickness of the created nonwovens was calculated as an average thickness measure on the side view SEM image, respectively $3.77 \mu \mathrm{m}$ and $3.89 \mu \mathrm{m}$.

\section{Electrospinning Process}

The nanofiber layers were prepared from polymeric solutions with a commercially available NanoSpider ${ }^{\mathrm{TM}}$ machine (Elmarco s.r.o. Liberec, Czech Republic, http://www.elmarco.com/) [8-9] equipped with patented rotating electrode with 3 cotton cords spinning elements (PCT/CZ2010/000042). The experimental conditions were as follows: relative humidity $25 \%$, temperature $24^{\circ} \mathrm{C}$, electric voltage applied into PU solution $75 \mathrm{kV}$, distance between electrodes $210 \mathrm{~mm}$, rotational electrode speed $7 \mathrm{rpm}$ and speed of supporting textile collecting nanofibers was $0.32 \mathrm{~m} \cdot \mathrm{min}^{-1}$. Nanofibers were collected on viscose nonwoven textiles (NT). 


\section{Filtration Efficiency}

The filtration efficiency in the ultrafine particle size range was determined as a function of particle diameter as shown in Figure 1. A $1 \mathrm{~g} / 1$ ammonium sulphate solution was nebulised (AGK, PALAS, Germany), a monodisperse size fraction was selected using an Electrostatic Classifier (EC 3080, TSI, USA), and particle concentration upstream and downstream the filter (face velocity $5.7 \mathrm{~cm} . \mathrm{s}^{-1}$ ) was recorded by a condensation particle counter (UCPC 3025 A, TSI, USA). The filtration efficiency was determined at nine mobility diameter fractions: 20, 35, 50, 70, 100, 140, 200, 280 and $400 \mathrm{~nm}$.

Even the mass area and thickness of both samples were very similar, there were differences in filtration efficiency observed, as can be seen in the graph below (see Figure 1). The Maximum Penetration Pore Size (MPPS) is similar for both samples, approximately $70 \mathrm{~nm}$, but the minimum filtration efficiency is lower for sample 1 (88.4\%) in comparison with sample $2(91 \%)$ and simultaneously the measured average pressure drop over sample 2 was lower than over sample 1, $91 \mathrm{~Pa}$ and $109 \mathrm{~Pa}$, respectively. These both filtration characteristics results in quality factor, $Q F$, equal to $23.7 \mathrm{kPa}^{-1}$ and $22.1 \mathrm{kPa}^{-1}$ for sample 1 and sample 2, respectively, where $Q F$ is defined as $Q F=-\ln (P) / \Delta p$ where $\Delta p$ is the pressure drop and $P$ is the penetration parallel to MPPS. Each filtration efficiency values and its pressure drops were measured repeatedly at steady state of experimental lay out.

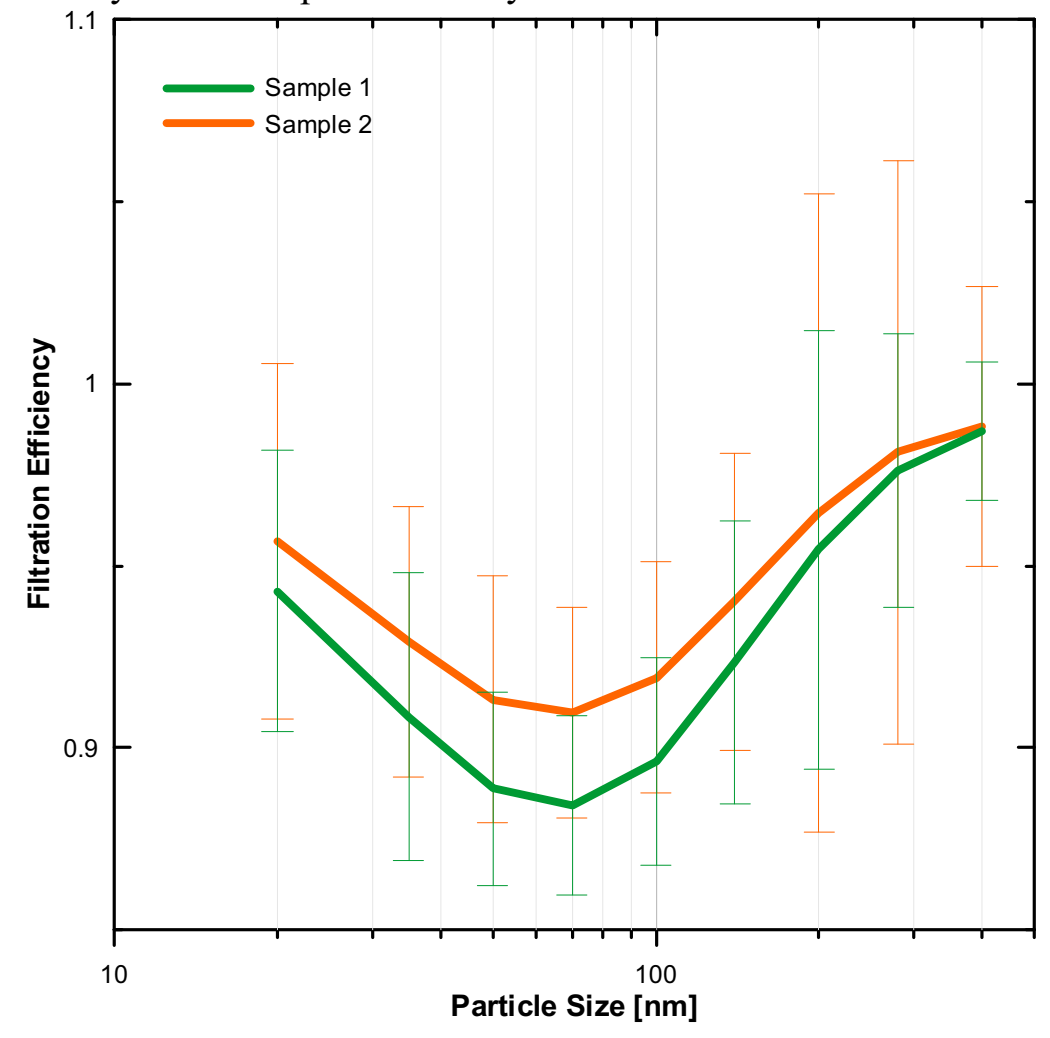

FIGURE 1. Filtration Efficiency Curve. 


\section{Electrostatic Characteristics}

The electric potential on both nanofiber based filters was measured by electrostatic fieldmeter Simco, model FMX-003, the Netherlands. It has been found that the difference in electric potential, $70 \mathrm{~V}$ of Sample 1 and $90 \mathrm{~V}$ for Sample 2, was negligible. Thus it can be concluded that the measured filtration characteristics depicted in Figure 1 for both samples were not influenced by any possible electrical charge on the polymeric nanofibers surface.

\section{Morphology Characterization}

In order to measure the basic morphological characteristics, the pore size distribution [9] and the fiber diameter distribution were measured for the nonwovens created by each polymer solution. In order to do this, SEM images for sample 1 and sample 2 prepared from solution A and B, respectively, (see Figure 2) were used. The morphological characteristics for both samples are provided in Figure 3. It is clearly visible that even if the average fiber diameter is almost the same for both samples sample 1 contains larger pores than sample 2.
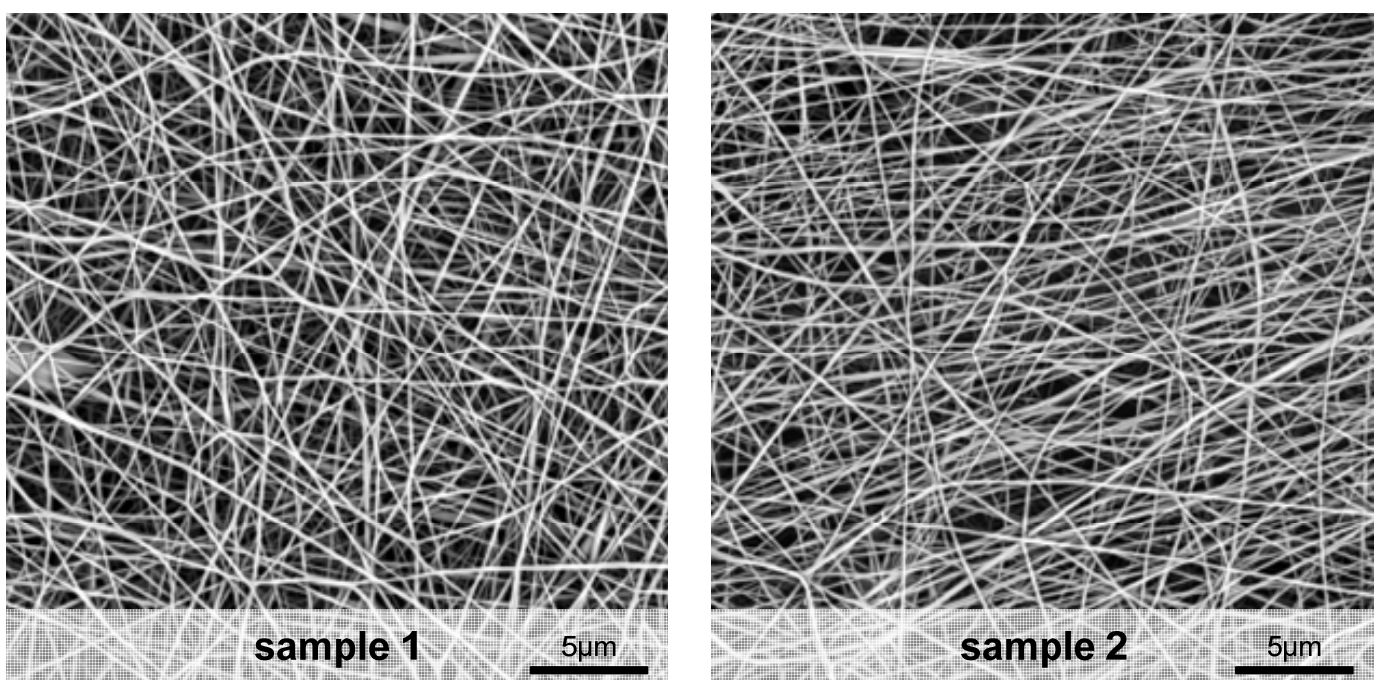

FIGURE 2. SEM images of the toplayers of samples 1 and 2 (electrospun fibers from solution A and B). 


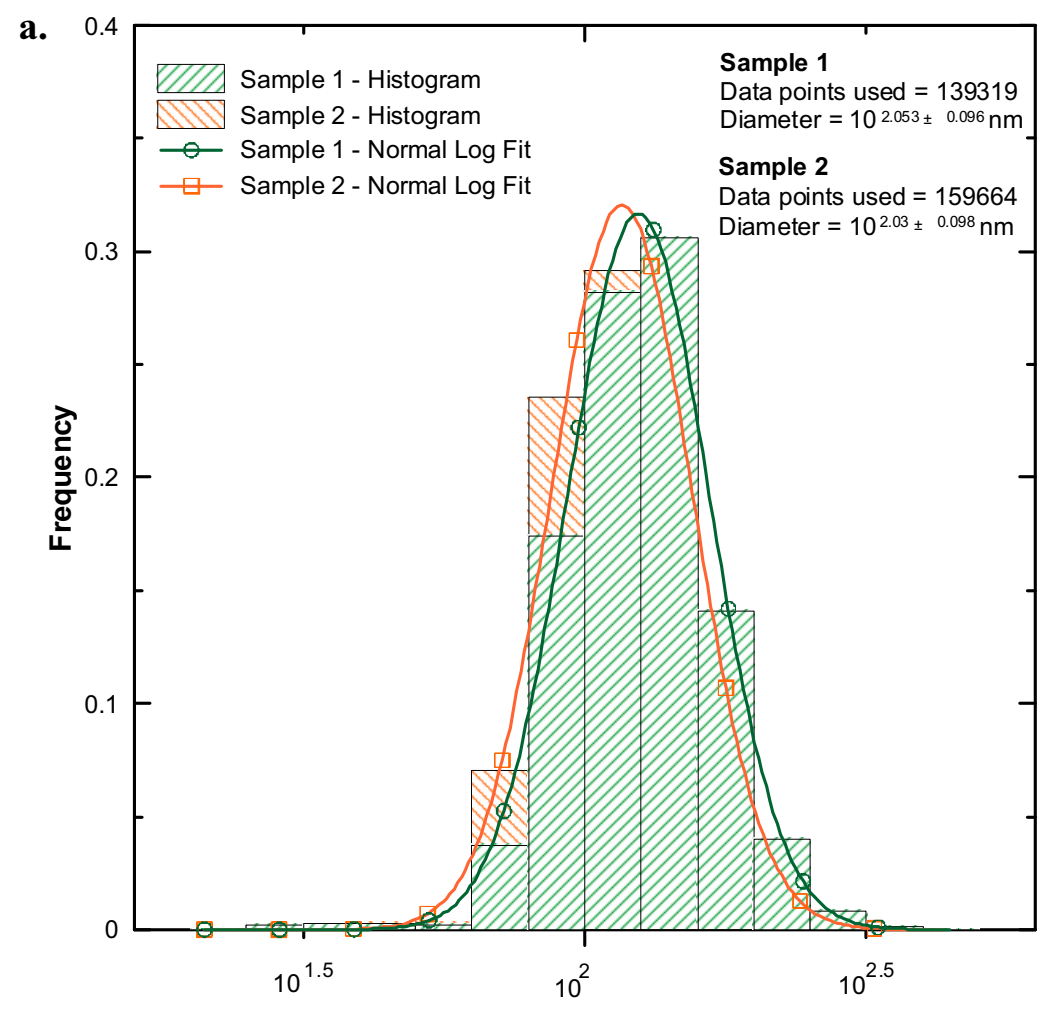

Fiber Diameter [nm]

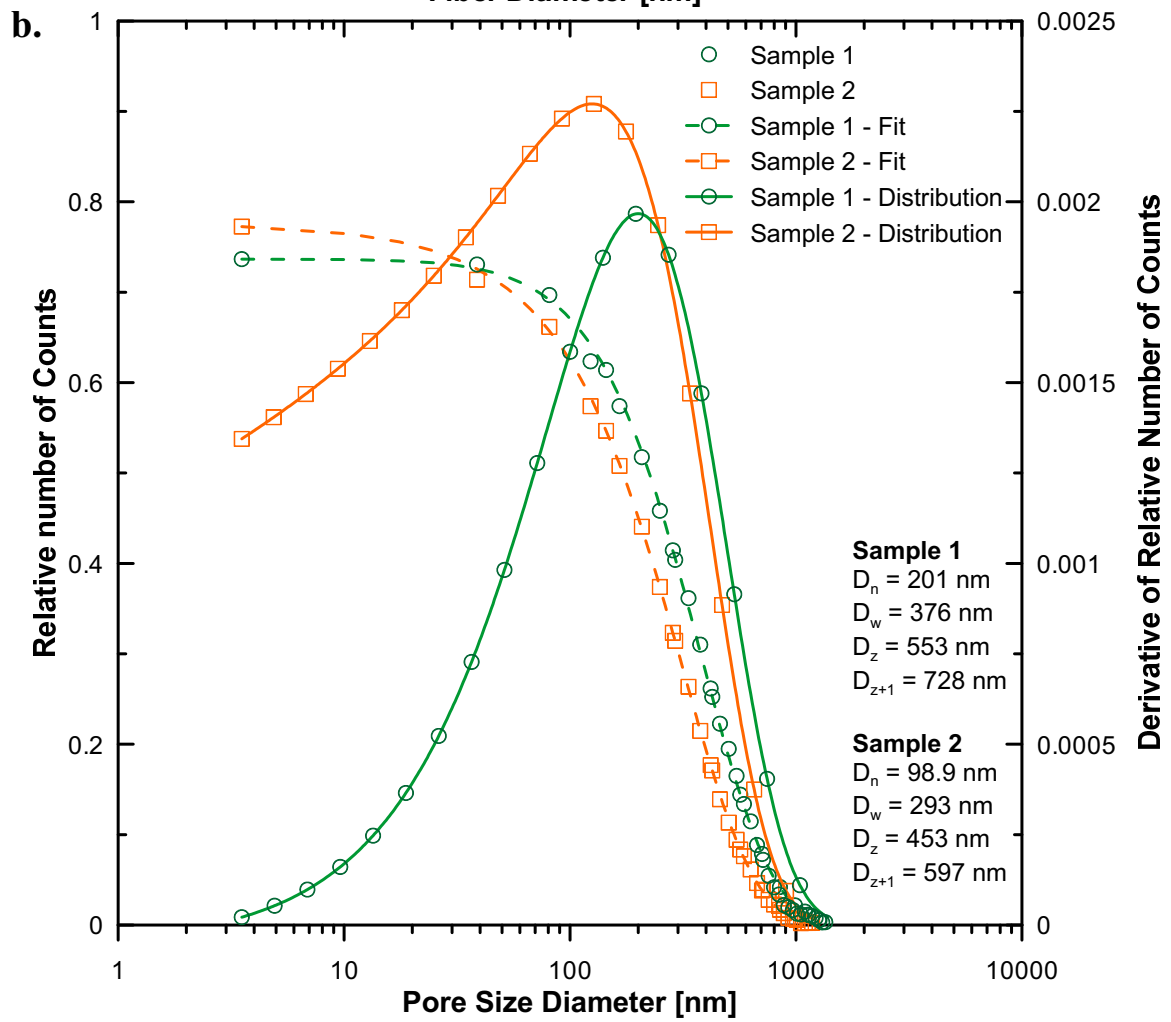

FIGURE 3. Fiber diameter distribution (a) and poresize distribution (b) of sample 1 and 2. 


\section{THEORETICAL}

\section{D Structure Model Creation}

Three dimensional model of the prepared nanofiber based filter has been created from SEM sample images, mass area and thickness in the following way (see Figure 4). In the first step the average threshold level has been applied for the original grayscale SEM sample image to filter the top fibers only, which results in a black/white image. In the second step, nanofiber centerlines have been calculated according the algorithm developed by Huang et al. [10]. In the following step, circles with centerpoint, a point of the centerline, have been fitted in the white fiber area and consequently rotated along the fiber centerline which results in a single $3 \mathrm{D}$ model layer and can be used to create the full 3D model having given mass area and thickness. It should be mentioned that in our previous work [8], the fiber structure complexity has been reduced considerably due to assumption that each nanofiber can be viewed as the number of interconnected tubes. In this work, this assumption is relaxed in order to take varying fiber, curvature and possible defect correctly into account. The comparison between the 'old methodology' [8] and the 'improved methodology' utilized in this work is provided in Figure 5 for an identical SEM image. It is clearly visible that the improved methodology allows representing the 3D nanofiber based structure more realistically than utilization of old methodology.

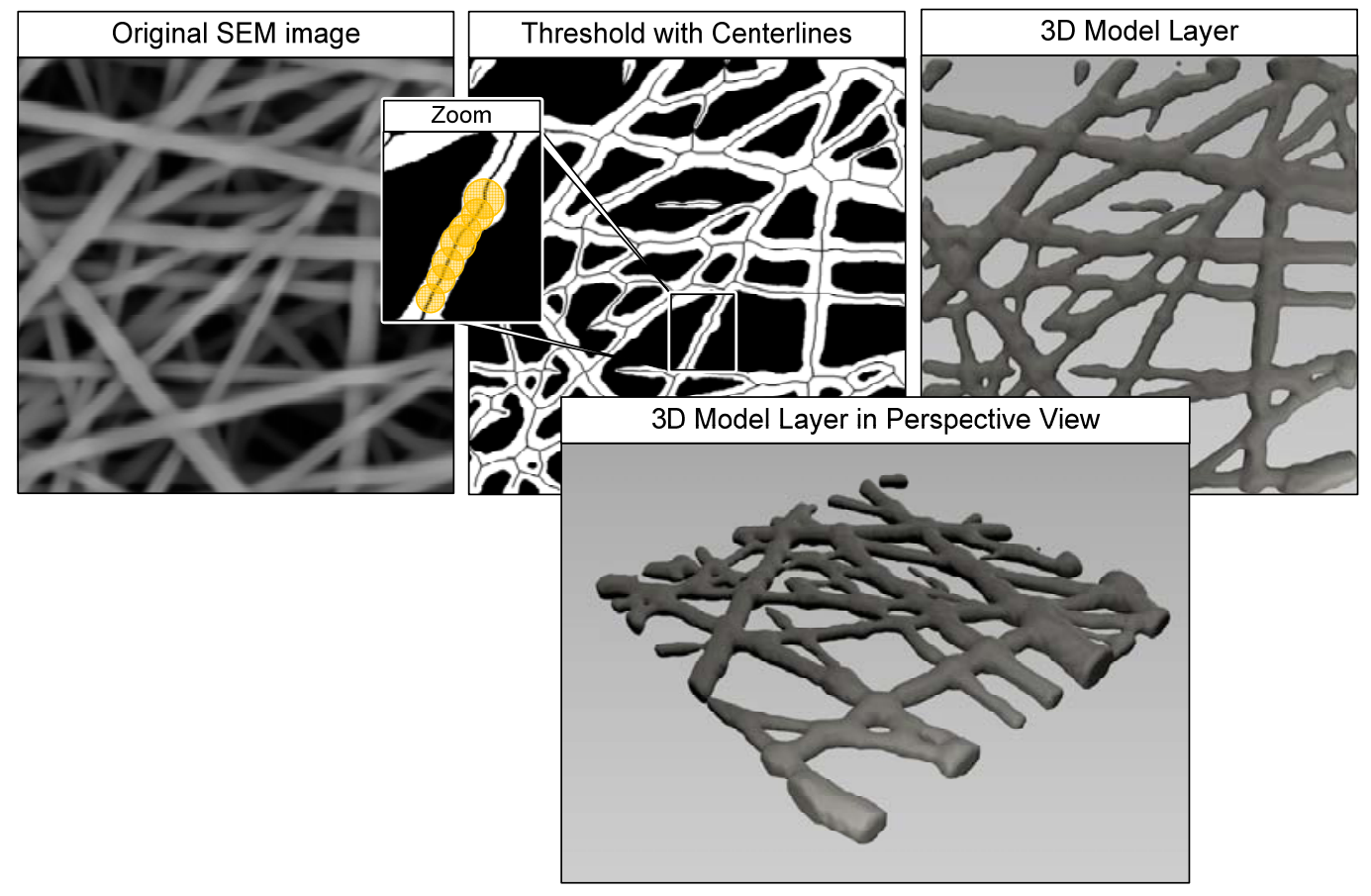

FIGURE 4. Basic principal of the 3D model layer generation from a SEM image. 


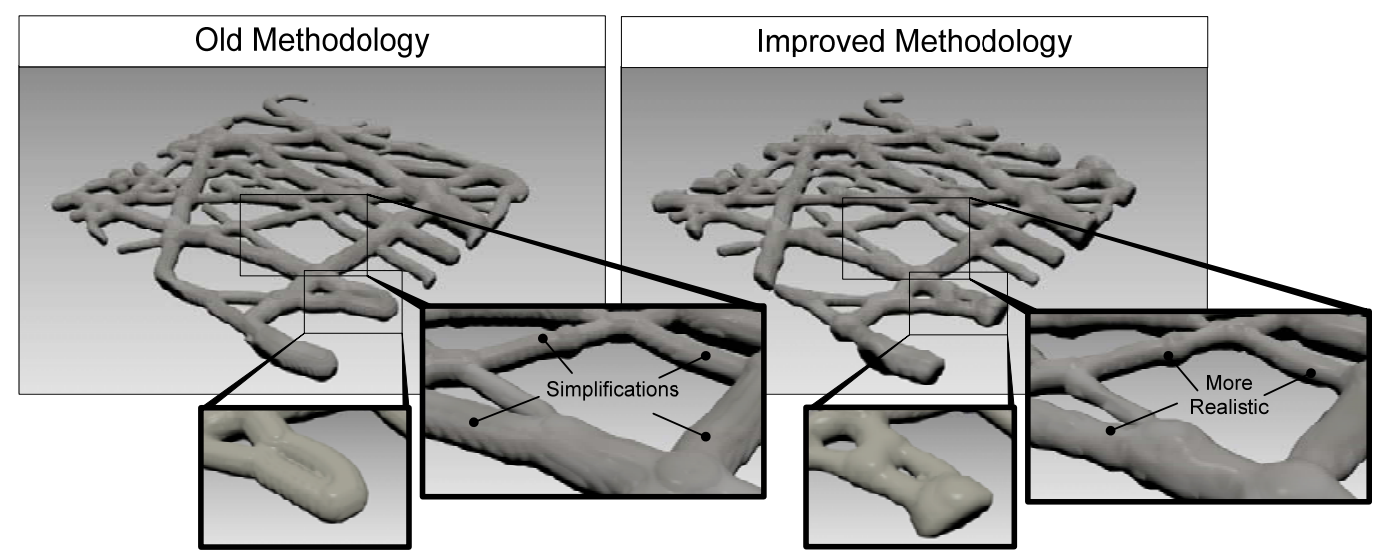

FIGURE 5. Comparison between the 3D nanofiber structure model created from SEM image by using old and improved methodology.

Considering a final sample thickness as well as the fact that a single hydrophobic layer of nanofibers prepared from solution A has a mass area of 0.1095 g.m ${ }^{-2}$ and the hydrophilic layer of nanofibers prepared from solution has a mass area of $\mathrm{B}$, 0.3372 g.m ${ }^{-2}$, full 3D models of sample 1 and sample 2 can be created (see Figure 6 ).

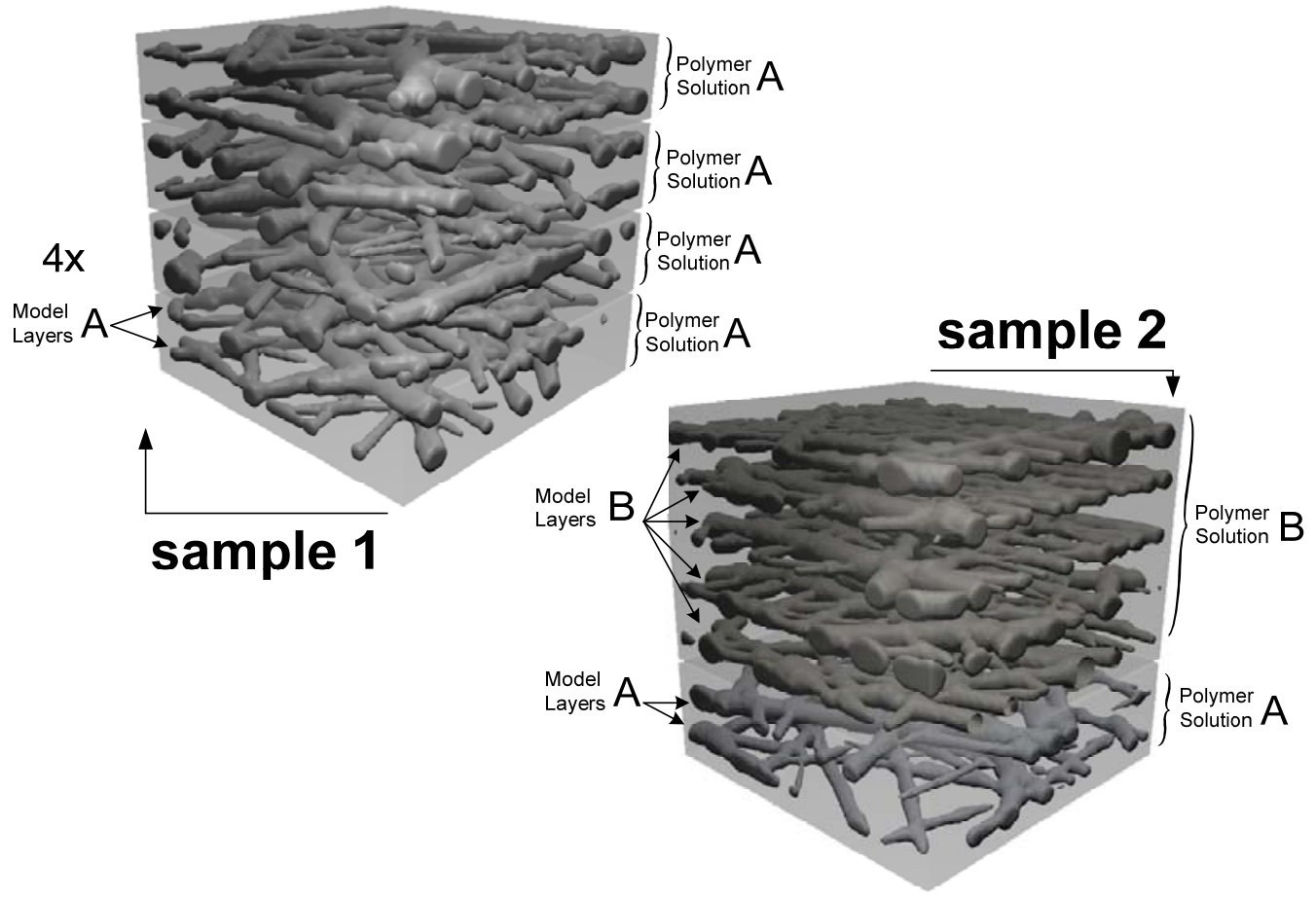

FIGURE 6. 3D nanofiber structure models for sample 1 and sample 2. 


\section{Theoretical Filtration Model}

The filtration model which is used in this work was introduced in our previous work $[8,9]$. In more detail, uniform distribution of nanoparticles is generated and penetrate the nanofiber nonwoven model according a simulated Brownian diffusion path. In order to calculate the Brownian motion position at each time step the following equations [5] are utilized for two intervals randomly divided into the given time step by a single random coefficient $U \in[0 ; 1[$ to shift the Brownian motion sampling. For a time interval $[\mathrm{j} \Delta \mathrm{t} ;(\mathrm{j}+\mathrm{U}) \Delta \mathrm{t}]$ :

$$
\begin{gathered}
v_{\mathrm{i}_{\mathrm{p}}}((j+U) \Delta t)=\left(v_{\mathrm{i}_{\mathrm{p}}}(j \Delta t)-v_{\mathrm{i}_{\mathrm{f}}}-\frac{F_{\mathrm{b}_{\mathrm{i}}}}{\beta}\right) e^{(-\beta U \Delta t)}+\frac{F_{\mathrm{b}_{\mathrm{i}}}}{\beta}+v_{\mathrm{i}_{\mathrm{f}}}, \\
x_{\mathrm{i}_{\mathrm{p}}}((j+U) \Delta t)=\left(\frac{v_{\mathrm{i}_{\mathrm{p}}}(j \Delta t)-v_{\mathrm{i}_{\mathrm{f}}}-\frac{F_{\mathrm{b}_{\mathrm{i}}}}{\beta}}{\beta}\right)\left(e^{(-\beta U \Delta t)}-1\right)+\left(\frac{F_{\mathrm{b}_{\mathrm{i}}}}{\beta}+v_{\mathrm{i}_{\mathrm{f}}}\right) U \Delta t+x_{\mathrm{i}_{\mathrm{p}}}(j \Delta t),
\end{gathered}
$$

and time interval $[(\mathrm{j}+\mathrm{U}) \Delta \mathrm{t} ;(\mathrm{j}+1) \Delta \mathrm{t}]$ :

$$
\begin{gathered}
v_{\mathrm{i}_{\mathrm{p}}}((j+1) \Delta t)=\left(v_{\mathrm{i}_{\mathrm{p}}}((j+U) \Delta t)-v_{\mathrm{i}_{\mathrm{f}}}-\frac{F_{\mathrm{b}+1}}{\beta}\right) e^{(-\beta(1-U) \Delta t)}+\frac{F_{\mathrm{b}_{\mathrm{i}+1}}}{\beta}+v_{\mathrm{i}_{\mathrm{f}}}, \\
x_{\mathrm{i}_{\mathrm{p}}}((j+1) \Delta t)=\left(\frac{v_{\mathrm{i}_{\mathrm{p}}}((j+U) \Delta t)-v_{\mathrm{i}_{\mathrm{f}}}-\frac{F_{\mathrm{b}_{i+1}}}{\beta}}{\beta}\right)\left(e^{(-\beta(1-U) \Delta t)}-1\right)+\left(\frac{F_{\mathrm{b}_{i+1}}}{\beta}+v_{\mathrm{i}_{\mathrm{f}}}\right)(1-U) \Delta t+x_{\mathrm{i}_{\mathrm{p}}}((j+U) \Delta t)
\end{gathered}
$$

where $v_{\mathrm{i}_{\mathrm{f}}}$ and $v_{\mathrm{i}_{\mathrm{p}}}$ are the fluid and particle velocities in the $\mathrm{x}, \mathrm{y}$, or $\mathrm{z}$ direction,

$$
\beta=\frac{18 \eta}{d_{\mathrm{p}}^{2} \rho_{\mathrm{p}} C_{\mathrm{c}}},
$$

is the relaxation frequency of the particle, $d_{\mathrm{p}}$ and $\rho_{\mathrm{p}}$ are the particle diameter and density, $\eta$ is liquid viscosity, $C_{\mathrm{c}}=1+K_{\mathrm{n}}\left(1.275+0.4 e^{\frac{-1.1}{K_{\mathrm{n}}}}\right)$ is an empirical Cunningham slip correction factor, $K_{\mathrm{n}}$ is the fiber Knudsen number

$$
K_{\mathrm{n}}=\frac{2 \lambda}{d_{\mathrm{f}}}
$$

where $d_{\mathrm{f}}$ is the fiber diameter and $\lambda$ is the mean free path of gas molecules

$$
\lambda=\frac{R T}{\sqrt{2} N_{\mathrm{A}} \pi d_{\mathrm{m}}^{2} p}
$$


where $R$ is the gas constant, $T$ the temperature, $N_{\mathrm{A}}$ number of Avogadro, $p$ the pressure and $d_{\mathrm{m}}$ Collision diameter of an air molecule $\left(3.7 .10^{-10} \mathrm{~m}\right)$.

$F_{\text {bi }}$ is the Brownian white noise excitation due to the impact of air molecules on the particle defined according to [11-13] as

$$
F_{\mathrm{b}_{\mathrm{i}}}=\varsigma_{\mathrm{i}} \sqrt{\frac{\pi S_{\mathrm{nn}}}{\Delta t}},
$$

where $\varsigma_{\mathrm{i}}$ are zero-mean, unit-variance independent Gaussian random numbers, $S_{\mathrm{nn}}$ is the spectral intensity (characterizing the noise intensity) defined as

$$
S_{\mathrm{nn}}=\frac{3 k T \beta}{\pi d_{\mathrm{p}}^{3} \rho_{\mathrm{p}}}
$$

where $k$ is the Boltzmann constant and $T$ the temperature.

When a particle get in contact with a fiber the particle can slip around the fiber or stick. In order to decide this, a force balance based on the balance suggested by Altmann and Ripperger [14], between the particle and the fiber is evaluated. In this force balance it is considered that the flow through a pore can be viewed as a Poiseuille flow in a 2D duct. The gas viscosity is reduced due to slip and corrected at high Knudsen numbers $\left(K_{\mathrm{n}}>0.01\right)$. For this correction the equations proposed by McNenly et al. [15] has been utilized for Poiseuille flows:

$$
\eta=\eta^{\prime}\left[a_{0}+a_{1} \arctan \left(a_{2} K_{\mathrm{n}}^{a_{3}}\right)\right]
$$

where $\eta^{\prime}$ is the gas viscosity at given pressure and temperature, $a_{0}=1.066, a_{1}=0.679$, $a_{2}=-2.082, a_{3}=0.866$.

In the force balance, the friction force, $F_{\mathrm{F}}$, is working against the drag-force, $F_{\mathrm{D}}$, and the lift-force, $F_{\mathrm{L}}$, is working against the adhesion force, $F_{\mathrm{A}}$. These forces are defined in the following way:

\section{Drag force, $F_{\mathrm{D}}$}

Drag force starts to act on the fiber intercepted particle due to pressure driven shear flow occurring inside the pore having average diameter $H$, because velocity between the solid particle and the fluid starts to be different. According to theoretical and experimental work of Rubin [16], Altman and Ripperger [14], the drag force can be expressed in the following way:

$$
F_{\mathrm{D}}=3.16 \pi \tau_{\mathrm{w}} d_{\mathrm{p}}^{2},
$$

where $\tau_{\mathrm{w}}$ is local fiber wall shear stress, which can be calculated from the Newtonian law as

$$
\tau_{\mathrm{w}}=\eta \dot{\gamma},
$$

where $\dot{\gamma}$ represents shear rate at the fiber wall. Note that the characteristic pore size dimension $H$ is determined here as the perpendicular distance between the interception particle/fiber point and surrounding fiber, which represents the second part of the pore. 


\section{Lift force, $F_{\mathrm{L}}$}

According to the theoretical and experimental analysis of Rubin [16] and [14, 17], the lift force is caused by the shear flow and can be calculated as follows:

$$
F_{\mathrm{L}}=\frac{0.761 \tau_{\mathrm{w}}^{1.5} d_{\mathrm{p}}^{3} \rho_{\mathrm{f}}^{0.5}}{\eta},
$$

where $\rho_{\mathrm{f}}$ is density of the fluid.

\section{Adhesive force $F_{\mathrm{A}}$}

The estimation of the this force is complicated because different parameters such as particle/fiber shape, particle/fiber roughness, adhesion distance, number of contact points etc. may influence the adhesive force strongly [14]. Neglecting the electrostatic interactions, the adhesive forces can be evaluated by calculating van der Waals force between two ideal spheres as follows:

$$
F_{\mathrm{A}}=\frac{\hbar \bar{\omega} d_{\mathrm{p}}}{32 \pi a^{2}}
$$

where $\hbar \bar{\omega}$ is the Lifschitz-van der Waals constant $\left(10^{-20} \mathrm{~J}\right)$ and $a$ is the adhesive distance $(0.4 \mathrm{~nm}[4,14])$.

\section{Friction force $F_{\mathrm{F}}$}

The friction force is caused by the action of the forces acting towards the fiber surface (van der Waals force $F_{\mathrm{A}}$ in this case).

$$
F_{\mathrm{F}}=\mu F_{\mathrm{A}}
$$

where $\mu$ is the friction coefficient $(0<\mu<1)$.

The particle is considered to be intercepted by the fiber when the lift force is lower/equal than the adhesion force, $F_{\mathrm{L}} \leq F_{\mathrm{A}}$, or the drag force is lower/equal than the friction force, $F_{\mathrm{D}} \leq F_{\mathrm{F}}$. On the other hand, if the lift force is higher than the adhesion force $\left(F_{\mathrm{L}}>F_{\mathrm{A}}\right)$ or the drag force is higher than the friction force $\left(F_{\mathrm{D}}>F_{\mathrm{F}}\right)$, the particle starts to slip around the fiber surface until it reach the mirrored position on this fiber. When the particle has two or more connection points with the fiber then the particle will also be caught, due to the higher attraction between particle and fiber.

Additionally, the sieve effect is taken into account and for bigger particles this effect is more pronounced. The sieve effect occurs when the particle cannot slip through two fibers.

Due to the improvement of the 3D nanofiber structure model, the slip path around the fibers cannot be seen as a simple circular path, as was done in our previous work [8], but more general path which follows the tube with varying shape, has to be considered and utilized. 


\section{RESULTS AND DISCUSSION}

\section{Filtration Efficiency Simulation}

The following parameters were used for the filtration efficiency simulation purposes: the friction coefficient between the particle and fiber equal to 0.002 , air viscosity equal to $2.910^{-5} \mathrm{~Pa} . \mathrm{s}$, the ammonium sulphate particles density equal to $1000 \mathrm{~kg} . \mathrm{m}^{-3}$, the air velocity $0.057 \mathrm{~m} \cdot \mathrm{s}^{-1}$ (which is the same as during the filtration efficiency measurements). Hundred groups of particles having the size between 20 and $500 \mathrm{~nm}$ (each diameter set consist of 1000 particles) were utilized. The predicted filtration efficiency data for both samples were averaged by using a moving average in order to reduce the noise. Comparison between the measured and predicted filtration efficiency characteristics for both samples is provided in Figure 7. It is nicely visible that the model predictions are in good agreement with the corresponding experimental data which justifies all assumptions in the used filtration model as well as the way of 3D nanofiber based structure model creation from SEM images.

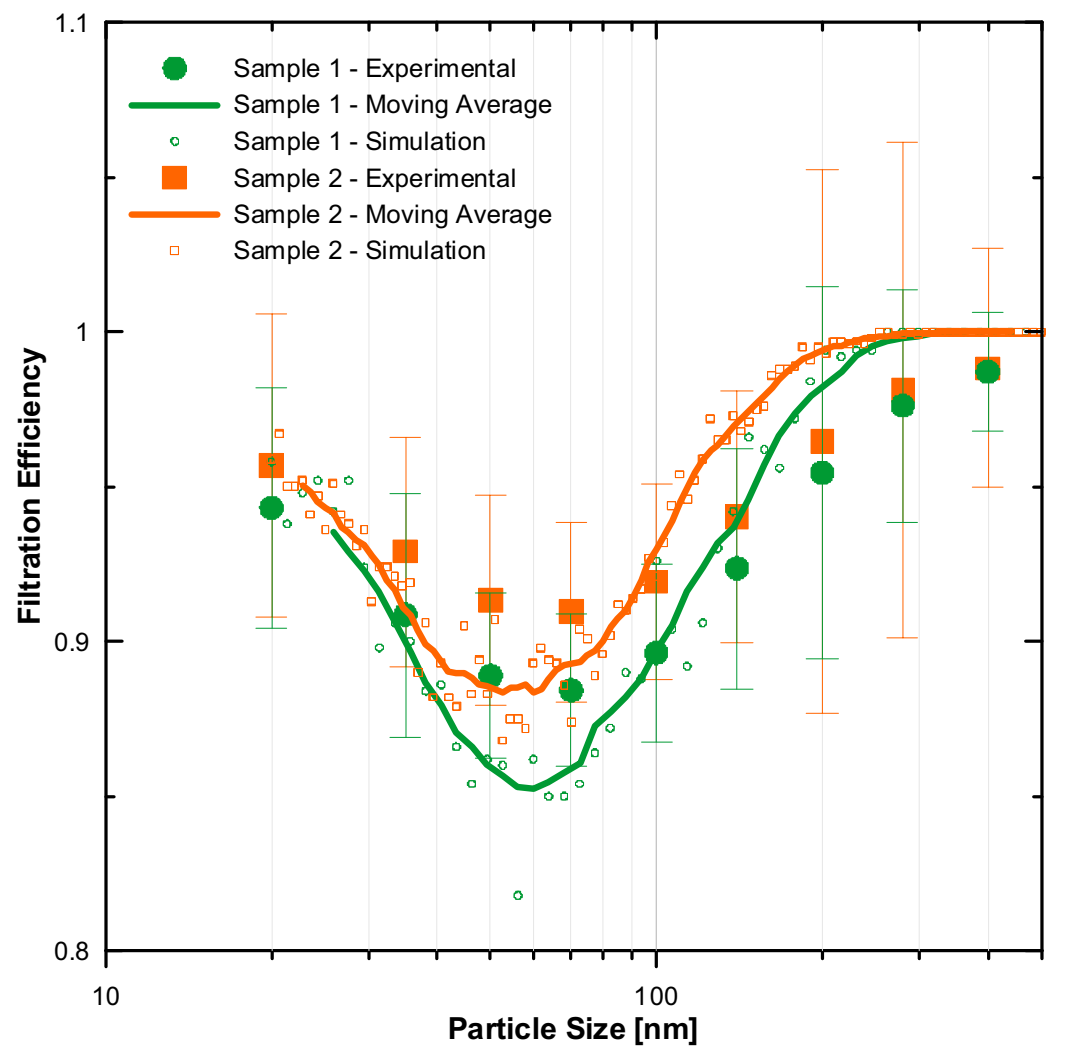

FIGURE 7. Comparison between experimental and simulation results of sample 1 and 2. 


\section{Influence of the Friction Coefficient on the Filtration Efficiency}

In this part, different virtual 3D nanofiber based structures consisting of different nanofiber layers prepared from solution A and B where investigated in order to reveal the effect of the filter composition on its final filtration efficiency. In more detail, the following virtual structures (with friction coefficient for model layer $\mathrm{A}$ and $\mathrm{B}, 0.005$ and 0.01 , respectively) have been created: A-A-A, B-A-A, B-B-A and B-B-B.

The predicted filtration efficiency characteristics for all investigated structure combinations are provided in Figure 8. It can be seen, that the addition of one advanced structure (B layer) at the top of the filter, having less efficient layer A at the bottom, can increase the filtration efficiency significantly.

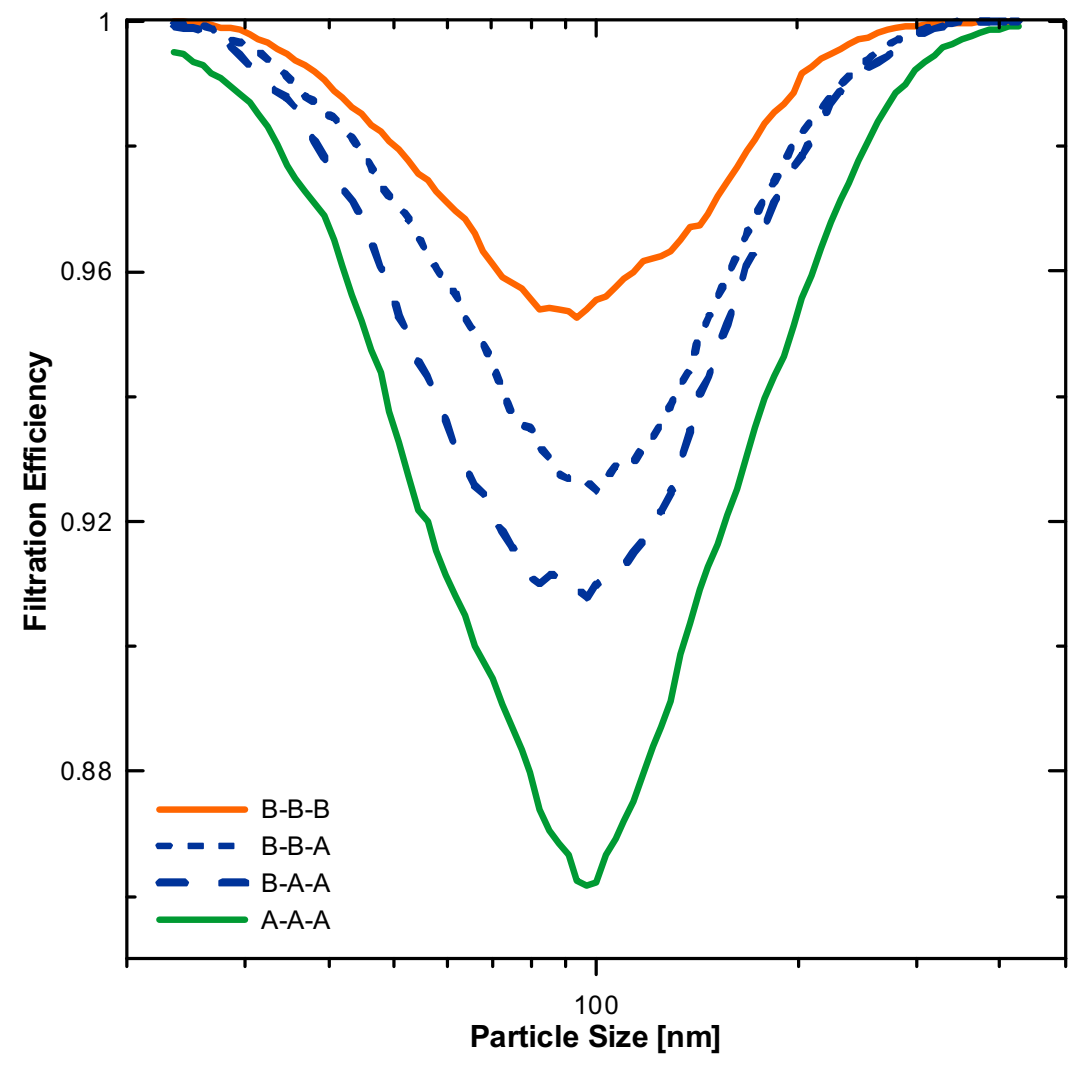

FIGURE 8. Effect of different nanofiber nonwovens on the filtration efficiency.

In the second part of the theoretical analysis, the effect of the friction coefficient between the polymeric nanofiber and the filtered particle has been investigated for a three layer structure A-A-A. In this structure, the friction coefficient of bottom layers 2 and 3 was adjusted to be 0.005 whereas the friction coefficient of the top nanofiber layer 1 was varying between 0.07 and 0.1 . The predicted filtration efficiency for all investigated combinations is depicted in Figure 9. As can be seen, increase of the friction coefficient of the top surface nanofiber layer increase the overall filtration efficiency of the filter considerably. This suggests that the nanofiber surface 
modification (at least the top layer) can enhance nanofiber filter efficiency considerably.

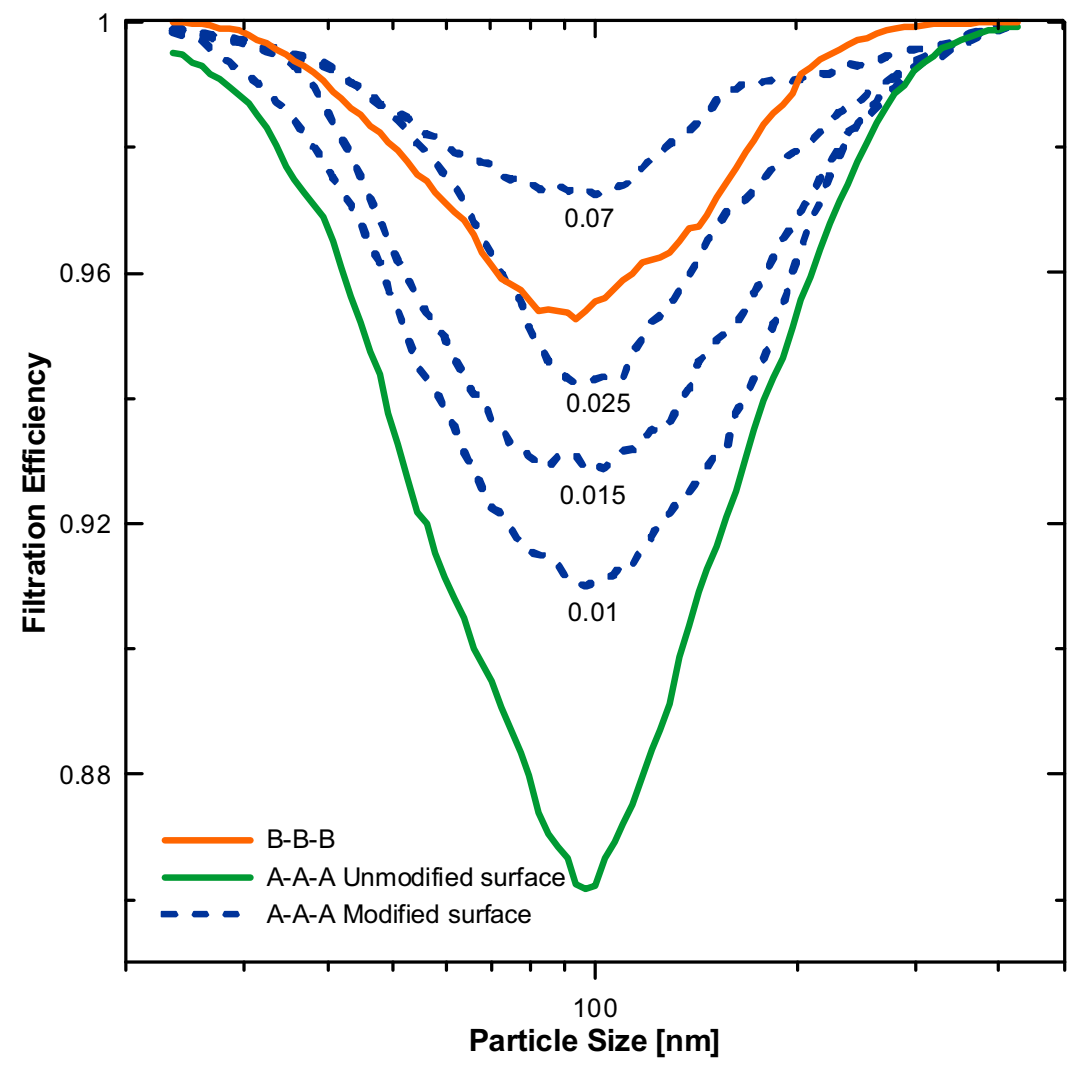

FIGURE 9. The effect of surface modification by changing the friction of the top layer on the filtration. efficiency.

\section{CONCLUSIONS}

In this work, novel 3D filtration model for nanofiber based filters has been proposed and tested. For the model validation purposes, filtration efficiency characteristics of two different polyurethane nanofiber based structures (prepared by the electrospinning process) were determined experimentally in the ultrafine particle size range $(20-400 \mathrm{~nm})$. It has been found that the proposed model is able to reasonably predict the measured filtration efficiency curves for both tested samples. The consequent theoretical analysis has revealed that addition of one advanced nanofiber structure layer at the top of the filter, having less efficient nanofiber layers at the bottom, can increase the filtration efficiency significantly. 


\section{ACKNOWLEDGMENTS}

The authors wish to acknowledge Grant Agency of the Czech Republic (grant No. P108/10/1325) and the Ministry of Education, Youth and Sports of the Czech Republic (MSM 7088352101) for the financial support. This article was written with support of Operational Program Research and Development for Innovations co-funded by the European Regional Development Fund (ERDF) and national budget of Czech Republic, within the framework of project Centre of Polymer Systems (reg. number: CZ.1.05/2.1.00/03.0111).

\section{REFERENCES}

1. Z. M. Huang, Y. Z. Zhang, M. Kotaki, et al., Compos. Sci. Technol. 63, 2223-2253 (2003).

2. A. L. Andrady, Science and Technology of Polymer Nanofibers, New Jersey: Wiley-Interscience, 2008, pp.404.

3. I. M. Hutten, Handbook of Nonwoven Filter Media, Burlington: Elsevier, 2007, pp.473.

4. R. C. Brown, Air Filtration: An Integrated Approuch to the Theory and Applications of Fibrous Filters, New York: Pergamon Press, 1993, pp.272.

5. B. Maze, H. V. Tafreshi, Q. Wang and B. Pourdeyhimi, J. Aerosol Sci. 38, 550-571 (2007).

6. S. A. Hosseini, H. V. Tafreshi, Chem. Eng. Sci. 65, 2249-2254 (2010).

7. S. A. Hosseini, H. V. Tafreshi, Powder Technology 201, 153-160 (2010).

8. W. Sambaer, M. Zatloukal and D. Kimmer, Chem. Eng. Sci.66, 613-623 (2011).

9. W. Sambaer, M. Zatloukal and D. Kimmer, Polymer Testing 29, 82-94 (2010).

10. L. Huang, G. Wan and C. Liu, "An Improved Parallel Thinning Algorithm" in Proceedings of the Seventh International Conference on Document Analysis and Recognition, 2003, pp.780-78

11. A. Li, G. Ahmadi, Aerosol Sc. Tech. 16, 209-226 (1992).

12. H. Ounis, and G. Ahmadi, Aerosol Sc. Tech. 13, 47-53 (1990).

13. H. Ounis, G. Ahmadi and J. B. Mclaughlin, J. Colloid Interf. Sci. 147, 233-250 (1991).

14. J. Altmann, S. Ripperger, J. membrane Sci. 124, 119-128 (1997).

15. M. J. McNenly, M. A. Gallis and I. D. Boyd, Int. J. Numer. Meth. Fl. 49, 1169-1191 (2005).

16.G. Rubin, "Widerstands und Auftreibsbeiwerte von ruhenden kugelformigen Partikeln in stationaren, wandnahen laminaren Grenzschichten", Ph.D. Thesis, TH Karlsruhe, 1977.

17. J. B. McLaughlin, J. Fluid Mech. 246, 249-265 (1993). 
Copyright of AIP Conference Proceedings is the property of American Institute of Physics and its content may not be copied or emailed to multiple sites or posted to a listserv without the copyright holder's express written permission. However, users may print, download, or email articles for individual use. 